\title{
Alberta Stroke Program Early CT Score in the Prognostication after Endovascular Treatment for Ischemic Stroke: A Meta-analysis
}

\author{
Chang-Woo Ryu, MD, PhD', Hee Shup Shin, MD, PhD², Soonchan Park, MD, PhD', \\ Sang Hyun Suh, MD, PhD, Jun Seok Koh, MD, PhD², Hye-Yeon Choi, MD, PhD³
}

Purpose: The Alberta Stroke Program Early CT Score (ASPECTS) was devised to quantify the extent of early ischemic changes in the middle cerebral artery territory on brain CT. We performed a systematic review and meta-analysis of studies that presented clinical outcomes and baseline ASPECTS in ischemic stroke patients managed with endovascular methods to validate the use of ASPECTS for risk prognostication.

Materials and Methods: We searched the MEDLINE, EMBASE, and Cochran databases for observational or interventional studies that reported clinical outcomes and baseline ASPECTS in ischemic stroke patients treated with endovascular methods. Data were pooled to perform a meta-analysis for comparisons of clinical outcomes between high and low ASPECTS patients.

Results: A meta-analysis of 13 studies (six observational and seven interventional) revealed favorable outcomes ( $\mathrm{mRS}$ sore $0-2$ at 90 days) for high baseline ASPECTS (odds ratio=2.22; 95\% Cl: 1.74-2.86).

Conclusion: High ASPECTS is a predictor of favorable outcome after endovascular therapy for ischemic stroke.

Key Words : ASPECTS score; Stroke; Thrombectomy; Reperfusion therapy

Departments of ${ }^{1}$ Radiology, ${ }^{2}$ Neurosurgery and ${ }^{3}$ Neurology, Kyung Hee University Hospital at Gangdong, School of Medicine, Kyung Hee University, Seoul, South Korea

${ }^{4}$ Department of Radiology, Gangnam Severance Hospital, Yonsei University College of Medicine, Seoul, South Korea

Received January 22, 2016;

accepted after revision February 20, 2017.

Correspondence to: Chang-Woo Ryu, MD, Department of Radiology, Kyung Hee University Hospital at Gangdong, 892 Dongnam-ro, Gangdong-gu, Seoul 05278, Korea

Tel. 82.2.440.6186 Fax. 82.2.440.6932

E-mail: md.cwryu@gmail.com

This is an Open Access article distributed under the terms of the Creative Commons Attribution Non-Commercial License (http://creativecommons.org/licenses/by-nc/3.0) which permits unrestricted non-commercial use, distribution, and reproduction in any medium, provided the original work is properly cited.
Several recent randomized controlled trials (RCTs) that evaluated clinical outcomes of endovascular therapy for acute ischemic stroke (AIS) have shown the benefits of endovascular therapy over intravenous thrombolysis (IVT) [1-5]. They suggested it should be accepted as a primary treatment choice for large vessel occlusion in AIS.

Appropriate patient selection for endovascular thrombectomy is critical and continues to be refined. This selection criteria is based on the time-window and baseline brain imaging. It is also important to predict the clinical outcome because baseline infarct volume is a factor associated with improved functional outcomes by recanalization treatment $[6-8]$. 
The Alberta stroke program early CT score (ASPECTS) was devised to quantify early ischemic changes on baseline CT in patients with AIS of the anterior circulation. This 10 -point quantitative topographic CT scan score can provide volumetric estimates of the size of cerebral infarction of anterior circulation. In many clinical studies of IVT, Although current data is uncertain relative to ASPECTS providing exclusion criteria for IVT, ASPECTS is a reliable predictor of functional outcomes, having strong correlations with functional outcome, mortality, and symptomatic intracranial hemorrhage (sICH) $[9,10]$.

To our knowledge, although ASPECTS was originally designed for use with IVT, recent studies indicated its practicability for use with endovascular treatment [11-13]. However, previous studies in smaller cohorts are insufficient to clarify the predictability of baseline ASPECTS in endovascular therapy. Therefore, a comprehensive review is needed to validate the clinical role of ASPECTS.

Our aim was to perform a comprehensive review and meta-analysis of clinical trials that recorded baseline ASPECTS in AIS patients managed with the endovascular method to yield reliable estimates of outcome prognostication of this approach before endovascular therapy.

\section{MATERIALS AND METHODS}

\section{Search Strategy}

Meta-analyses were undertaken in accordance with guidelines for meta-analysis of observational studies in epidemiology (MOOSE) [14]. Two researchers (C.R., S.S.) identified all published observational or interventional studies that reported clinical outcomes according to baseline ASPECTS in acute ischemic stroke patients treated with endovascular management. We conducted a systematic literature review of PubMed, EMBASE, and Cochrane databases from January 2000 to January 2016. The following key words and entry terms analogous with these were used for searching in relevant combinations by using the Boolean operators OR and AND: "cerebrovascular accident," "stroke," "ischemia," "embolectomy," "endovascular," "intraarterial thrombectomy," and "thrombolysis". Following the search, articles were then screened by title and abstract for the aforementioned inclusion criteria. The references of included articles were assessed to identify additional potentially relevant studies. The search was restricted to human studies in English.

\section{Inclusion and exclusion criteria}

Studies were selected in the primary list as follows: any study that 1) involved AIS patients eligible for endovascular therapy; 2) reported over 20 cases undergoing endovascular therapy; 3) reported baseline ASPECTS of brain imaging modality in AIS patients; and 4) accurately described functional outcomes using a modified Rankin scale (mRS) at 90 days. We excluded studies 1) having duplicated data; 2) reporting mainly patients with posterior circulation stroke; 3 ) in which ASPECTS was graded based on perfusion imaging only, and 4) lacking appropriate data. A PRISMA flow diagram was used to illustrate the decision-making process regarding the studies.

\section{Data extraction}

Three reviewers (C.R., S.P., H.S.) independently extracted data from selected studies that fulfilled the inclusion and exclusion criteria using a standardized form, and all disagreements were resolved by consensus. The following data were collected: report characteristics (first author's name, journal, year of publication); study design (retrospective/prospective analysis, intervention trial or observation, name of cohort, single/two arms, single/multicenter), indication for endovascular therapy, treatment option (the administration of intravenous thrombolytic agent (IVT) before endovascular therapy, type of intra-arterial approach), study sample (sample size, age, sex, baseline National Institutes of Health Stroke Scale [NIHSS], baseline ASPECTS, the occlusive segment, time from symptom onset to recanalization, rate of recanalization); and data regarding and definitions of outcomes (favorable functional outcome, mortality, and sICH). If a study presented outcomes according to dichotomized ASPECTS, we used these data without modification, and if not, ASPECTS was dichotomized into high $(>7)$ and low $(\leq 7)$ using data extracted for primary analysis, as performed previously [15].

The quality of the included studies was also independently assessed by the two reviewers (C.R., H.S.) using the "Quality Assessment Tool for Observational Cohort and Cross-Sectional Studies," provided by the National Institutes of Health (NIH) [16] and/or Cochrane Collaboration's tool for assessing risk of bias [17].

\section{Outcome measures}

Predictability was assessed by comparisons of outcomes between dichotomized ASPECTS: high and low. The primary outcome of interest was the proportion of patients with favorable $\mathrm{mRS}$ scores of 0 to 2 at 


\section{Chang-Woo Ryu, et al.}

90 days post intervention. The secondary outcomes assessed were all-cause mortality and sICH. We calculated, and subsequently pooled in independent meta-analyses, the odds ratios (ORs) with corresponding $95 \%$ confidence intervals (CI) for each outcome of interest.

For all meta-analyses, outcomes were pooled using the DerSimonian and Laird random-effects model, with weights calculated by the Mantel-Haenszel method. Heterogeneity between studies was assessed using Cochrane's Q test and $\mathrm{I}^{2}$ statistic, with $\mathrm{I}^{2}$ values exceeding $50 \%$ representing significant heterogeneity. Publication bias was evaluated visually by funnel plots or quantified by the Begg-Mazumdar's rank correlation test.

Subgroup analyses and meta-regression were used to determine whether study-related factors could account for heterogeneity. Subgroup analysis was performed according to the study design and included intraarterial chemical thrombolysis as the primary endovascular treatment option versus mechanical thrombectomy as the primary option. We were able to analyze the impacts of the rate of internal carotid artery (ICA) occlusion, the rate of recanalization, and time from symptom to recanalization for meta-regressions. In order to evaluate the stability of the pooled results, we further conducted sensitivity analyses by removing studies with higher risks of introducing bias.

A further analysis was conducted with ASPECTS dichotomized at very low $(0-4)$ versus intermediate and

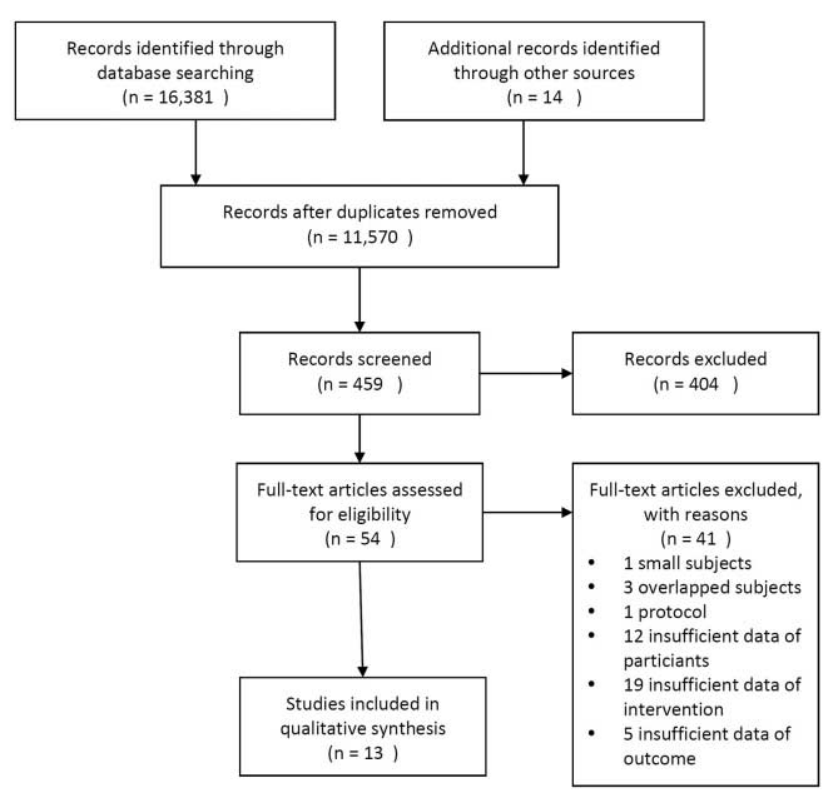

Fig. 1. PRISMA (Preferred Reporting Items for Systematic Reviews and Meta-Analyses) flow diagram describing selection process for included studies. high (5-10), and dichotomized at indeterminate ( 5 or $6-7)$ versus high (8-10). P-values $<0.05$ were considered significant.

\section{RESULTS}

\section{Study selection}

A total of 16,831 studies were identified during the initial search. Of these, 13 studies were pertinent to include in this review [3-5, 11-13, 18-24]. A flow diagram summarizing the literature search is presented in Figure 1.

\section{Study Characteristics}

The main methodological and baseline characteristics of included studies are presented in Tables 1 and 2. Three studies were primary reports of RCTs (ESCAPE, REVASCAT, SWIFT-PRIME) that compared the treatment effects of endovascular therapy to those of the current standard therapy for patients with AIS (3$5)$, and three studies were ancillary analyses of endovascular arms of RCTs (PROACT-II, DEFUSE-II, IMS-III) [18, 21, 23]. One study used pooled data from two single-arm trials (Penumbra phase II pivotal trial; ClinicalTrials.gov; NCT00334061, and Penumbra Imaging Collaborative Study; ClinicalTrials.gov; NCT00785161) to assess the safety and efficacy of endovascular devices [11]. The other six studies were retrospective reviews of consecutive single-/multicenter case series. No studies were considered to be seriously flawed as per the Quality Assessment Tool for Observational Cohort and Cross-Sectional Studies (Table 3).

All locations of ischemic strokes were within the anterior circulation distribution. Two studies included only MCA occlusion as inclusion criteria. Median/ mean NIHSS and ASPECTS were similar within each study. Of 1,798 participants (nine studies), 74\% had IVT before endovascular treatment, and in two studies, patients did not receive intravenous thrombolytic agents before endovascular treatment according to their study protocols. In four studies, intraarterial chemical thrombolysis was the primary choice of endovascular therapy or was given to over a half of the endovascular cohorts. Penumbra suction systems and/or stent retrievers were used as the primary choice for endovascular thrombectomy in nine studies. Eleven studies used nonenhanced CT to score ASPECTS and three studies used MRI. An ASPECTS dichotomy threshold of seven was used in 12 studies and a threshold of six was used in two studies. 


\section{Alberta Stroke Program Early CT Score in the Prognostication after Endovascular Treatment for Ischemic Stroke}

\section{Prognosis Predictability}

Primary outcome

Meta-analysis of pooled data from the 13 included studies revealed favorable functional outcomes $(\mathrm{mRS}$ sore $0-2$ at 90 days) in favor of high baseline ASPECTS (OR=2.227; 95\% CI: 1.735 to 2.859 ; $\mathrm{P}<0.0001$ ) (Fig. 2A). There was no significant hetero-

Table 1. Designs of Included Studies in the Meta-analysis and Characteristics of Endovascular Treatment

\begin{tabular}{|c|c|c|c|c|c|c|c|c|c|}
\hline \multirow[b]{2}{*}{ Author } & \multirow[b]{2}{*}{ Year } & \multicolumn{2}{|l|}{ Study design } & \multicolumn{4}{|c|}{ Characteristics of Endovascular Therapy } & \multirow[b]{2}{*}{$\begin{array}{c}\text { Recanalization } \\
\text { time, median } \\
\text { (IQR), min }\end{array}$} & \multirow[b]{2}{*}{$\begin{array}{c}\text { ICA } \\
\text { occlusion, } \\
n\end{array}$} \\
\hline & & Design & $\begin{array}{c}\text { Single/ } \\
\text { multicenter }\end{array}$ & $\begin{array}{l}\text { Location } \\
\text { in }\end{array}$ & $\begin{array}{l}\text { Pre- } \\
\text { ntervention } \\
\text { iv tPA (n) }\end{array}$ & $\begin{array}{l}\text { Endovascular } \\
\text { method for } \\
\text { primary choice }\end{array}$ & $\begin{array}{c}\text { Recanalization } \\
\text { with } \mathrm{TICl} \\
2 \mathrm{~b}-3, \mathrm{n}\end{array}$ & & \\
\hline $\begin{array}{l}\text { Hill }^{18} \\
\text { (PROACT-II) }\end{array}$ & 2003 & $\begin{array}{l}\text { Ancillary analysis of } \\
\text { randomized trials }\end{array}$ & multi & A & $\mathrm{N}$ & $\mathrm{iA}$ & 68 (TIMI 2-3) & NA & NA \\
\hline Imai ${ }^{19}$ & 2011 & $\begin{array}{l}\text { Retrospective observation } \\
\text { of case series }\end{array}$ & single & A (MCA only) & $\mathrm{N}$ & $\mathrm{iA}$ & 36 & NA & 0 \\
\hline Psychogios ${ }^{20}$ & 2013 & $\begin{array}{l}\text { Retrospective observation } \\
\text { of prospective registry }\end{array}$ & single & A (MCA only) & NA & Penumbra & $31 / 51$ & $\begin{array}{c}292 \\
(246-357)\end{array}$ & 0 \\
\hline Hill21 (IMS-III) & 2014 & $\begin{array}{l}\text { Ancillary analysis of } \\
\text { randomized trials }\end{array}$ & multi & $A-P(10)$ & $Y(434)$ & $\operatorname{Mix}(266 / 423)^{a}$ & NR & $334 c$ & NA \\
\hline Inoue ${ }^{22}$ & 2014 & $\begin{array}{l}\text { Retrospective observation } \\
\text { of prospective registry }\end{array}$ & single & A & $Y(148)$ & $\mathrm{iA}$ & 98 (TIMI 2-3) & NA & 59 \\
\hline $\begin{array}{l}\text { Yoo }{ }^{11} \text { (Penumbra } \\
\text { Pivotal + PICS) }\end{array}$ & 2014 & $\begin{array}{l}\text { Ancillary analysis of single- } \\
\text { arm trials }\end{array}$ & - multi & A & $Y(98)$ & Penumbra & $211(\mathrm{TICl} 2-3)$ & NA & 58 \\
\hline $\begin{array}{l}\text { Espinosa de } \\
\text { Rueda }^{12}\end{array}$ & 2015 & $\begin{array}{l}\text { Retrospective observation } \\
\text { of case series }\end{array}$ & single & A & $Y(67)$ & $\begin{array}{l}\text { Stentretriever \& } \\
\text { penumbra }\end{array}$ & NA & $\begin{array}{l}400.46 \\
(212.2)^{c}\end{array}$ & 29 \\
\hline $\begin{array}{l}\text { Goyal }^{3} \\
\text { (ESCAPE) }\end{array}$ & 2015 & $\begin{array}{l}\text { Primary reports of } \\
\text { randomized trials }\end{array}$ & multi & A & $Y(120)$ & Stentretriever & $113 / 156$ & $\begin{array}{c}241 \\
(176-359)\end{array}$ & $45 / 163$ \\
\hline $\begin{array}{l}\text { Jovin }^{4} \\
\text { (REVASCAT) }\end{array}$ & 2015 & $\begin{array}{l}\text { Primary reports of } \\
\text { randomized trials }\end{array}$ & multi & A & $Y(68)$ & Stentretriever & $67 / 102$ & $\begin{array}{c}223 \\
(170-312)\end{array}$ & $26 / 102$ \\
\hline $\begin{array}{l}\text { McTaggart23 } \\
\text { (DEFUSE-II) }\end{array}$ & 2015 & $\begin{array}{l}\text { Ancillary analysis of } \\
\text { randomized trials }\end{array}$ & multi & $A$ & $\mathrm{Y}(\mathrm{NA})$ & $\operatorname{Mix}(\mathrm{NA})$ & NA & NA & 24 \\
\hline $\begin{array}{l}\text { Saver }^{5} \\
\text { (SWIFT-PRIME) }\end{array}$ & 2015 & $\begin{array}{l}\text { Primary reports of } \\
\text { randomized trials }\end{array}$ & multi & $A$ & $Y(95)$ & Stentretriever & $73 / 83$ & $\begin{array}{c}252 \\
(190-300)^{b}\end{array}$ & $17 / 93$ \\
\hline Spiotta $^{13}$ & 2015 & $\begin{array}{l}\text { Retrospective observation } \\
\text { of prospective registry }\end{array}$ & single & $A$ & $\mathrm{Y}(\mathrm{NA})$ & $\begin{array}{l}\text { Stentretriever \& } \\
\text { penumbra }\end{array}$ & 113 & NA & NA \\
\hline $\mathrm{Kim}^{24}$ & 2016 & $\begin{array}{l}\text { Retrospective observation } \\
\text { of prospective registry }\end{array}$ & single & $A$ & $Y(104)$ & Stentretriever & 144 & $\begin{array}{c}280 \\
(223.5-352)\end{array}$ & 44 \\
\hline
\end{tabular}

Abbreviations: CT, computed tomography; DEFUSE-II, The Diffusion and Perfusion Imaging Evaluation for Understanding Stroke Evaluation II; ESCAPE, Evaluation Study of Congestive Heart Failure and Pulmonary Artery Catheterization Effectiveness; EVT, endovascular treatment; IMS-III; Interventional Management of Stroke III; MR CLEAN, The Multicenter Randomized Trial of Endovascular Treatment for Acute Ischemic Stroke in the Netherlands; N, No; NIHSS, National Institutes of Health Stroke Scale; NR, not reported; PROACT-II, Prolyse in Acute Cerebral Thromboembolism II; PICS, Penumbra Imaging Collaborative Study; REVASCAT, Randomized Trial of Revascularization With Solitaire FR Device Versus Best Medical Therapy in the Treatment of Acute Stroke Due to Anterior Circulation Large Vessel Occlusion Presenting Within 8 Hours of Symptom Onset; SWIFT, SOLITAIRETM with the intention for thrombectomy; iA, intra-arterial thrombolytic drug infusion through catheter; TICI, Thrombolysis in Cerebral Infarction; TIMI, Thrombolysis In Myocardial Infarction; MCA, middle cerebral artery; ICA, internal carotid artery; Y, yes; N, no; NA, not applicable

a: Combinations of more than one endovascular therapy were used in 128 patients in total 266 patients with intraarterial tPA infusion.

b: The data of 'recanalization time' of this study means time interval from symptom onset to first stent deployment.

c. mean (standard deviation) 


\section{Chang-Woo Ryu, et al.}

geneity among the included studies (Q-statistics, $\left.\mathrm{P}=0.12 ; \mathrm{I}^{2}=32.96 \%\right)$. Significant publication bias was not observed (Kendal's tau, 0.000; two-tailed $\mathrm{P}=1.00$ )
(Fig. 2B).

Subgroup analyses of the seven prospective trials $(\mathrm{OR}=2.154 ; 95 \%$ CI 1.685 to $2.754 ; \mathrm{P}<0.0001$; Q-

Table 2. Characteristics of Patients in Endovascular and Medical Treatment Arms

\begin{tabular}{|c|c|c|c|c|c|c|c|c|c|c|c|c|c|}
\hline \multirow[t]{2}{*}{ Author } & \multicolumn{6}{|c|}{ Characteristics of patients in endovascular arm } & \multicolumn{6}{|c|}{ Characteristics of patients in medical arm } & \multirow[b]{2}{*}{$\begin{array}{l}\text { ASPECTS } \\
\text { dichotomy }\end{array}$} \\
\hline & $\begin{array}{l}\text { No. of } \\
\text { patients }\end{array}$ & $\begin{array}{c}\text { High: } \\
\text { Low } \\
\text { ASPECTS }\end{array}$ & $\begin{array}{c}\text { male, } \\
\mathrm{n}\end{array}$ & $\begin{array}{l}\text { Age, } \\
\text { mean } \\
(S D), y\end{array}$ & $\begin{array}{c}\text { ASPECT, } \\
\text { median } \\
(\mathrm{IQR})\end{array}$ & $\begin{array}{c}\text { NIHSS, } \\
\text { median } \\
(\mathrm{IQR})\end{array}$ & $\begin{array}{l}\text { No. of } \\
\text { patients }\end{array}$ & $\begin{array}{c}\text { High: } \\
\text { Low } \\
\text { ASPECTS }\end{array}$ & $\begin{array}{c}\text { male, } \\
\mathrm{n}\end{array}$ & $\begin{array}{l}\text { Age, } \\
\text { mean } \\
(S D), y\end{array}$ & $\begin{array}{c}\text { ASPECT, } \\
\text { median } \\
(\mathrm{IQR})\end{array}$ & $\begin{array}{c}\text { NIHSS, } \\
\text { median } \\
(\mathrm{IQR})\end{array}$ & \\
\hline $\begin{array}{l}\text { Hill }^{18} \\
\text { (PROACT-III) }\end{array}$ & 105 & 46: 59 & NA & NA & NA & NA & 49 & $20: 29$ & NA & NA & NA & NA & $0-7,8-10$ \\
\hline Imai ${ }^{19}$ & 90 & & 58 & $73(10.5)$ & NA & $18(7-26)^{\mathrm{h}}$ & & & & & & & $\begin{array}{c}\text { MRI-ASPECTS } \\
(5-7,8-10)\end{array}$ \\
\hline Psychogios $^{a 20}$ & 48 & & 28 & $69(21-86)^{9}$ & $8(6.5-9)$ & $17.5(6)^{\mathrm{h}}$ & & & & & & & $0-7,8-10$ \\
\hline Hill|21 (IMS-III) & 434 & $247: 187$ & 218 & $69(23-89)^{9}$ & NA & $17(7-40)$ & 222 & $131: 91$ & 122 & $68^{9}$ & NA & 16 & $0-7,8-10$ \\
\hline Inoue 22 & 210 & & 94 & $70.4(16.8)$ & $6(4-8)$ & $16(11-21)$ & & & & & & & $\begin{array}{c}\text { MRI-ASPECTS } \\
(0-7,8-10)\end{array}$ \\
\hline Yoo ${ }^{11}$ & 249 & & 124 & $66.6(15.0)$ & $8(6-9)$ & $17(13-21)$ & & & & & & & $0-7,8-10$ \\
\hline $\begin{array}{l}\text { Espinosa de } \\
\text { Rueda }^{12}\end{array}$ & 150 & & 77 & $66.3(12.6)$ & $8(7-9)$ & $17(13-22)$ & & & & & & & $3-7,8-10$ \\
\hline $\begin{array}{l}\text { Goyal }^{3} \\
(\text { ESCAPE) }\end{array}$ & 153 & $128: 25$ & 79 & $71(60-81)^{i}$ & $9(8-10)$ & $16(13-20)$ & 151 & $122: 29$ & 71 & $70(60-81)$ & ) $9(8-10)$ & $17(12-20)$ & $0-7,8-10$ \\
\hline Jovin ${ }^{d 4}$ & 103 & $50: 53$ & 55 & $65.7(11.3)$ & $7(6-9)$ & $17(14-20)$ & 103 & $55: 48$ & 54 & $67.2(9.5)$ & $8(6-9)$ & $17(12-19)$ & $6-7,8-10$ \\
\hline $\begin{array}{l}\text { McTaggart'23 } \\
(\text { DEFUSE-II) }\end{array}$ & 74 & & NA & $67(15)$ & $7.6^{h}$ & $17(12-20)$ & & & & & & & $0-7,8-10$ \\
\hline $\begin{array}{l}\text { Saver }^{5}(\text { SWIFT- } \\
\text { PRIME) }\end{array}$ & T- 95 & $71: 24$ & 54 & $65.0(12.5)$ & $9(7-10)$ & $17(13-20)$ & 90 & $71: 19$ & 54 & $65.0(12.5)$ & $9(7-10)$ & $17(13-20)$ & $6-7,8-10$ \\
\hline Spiotta $^{13}$ & 149 & & 72 & $66.1(15.1)$ & $8(3-10)^{9}$ & $16.2(6.7)^{\mathrm{h}}$ & & & & & & & $1-6,7-10$ \\
\hline $\mathrm{Kim}^{24}$ & 171 & & 887 & $72(63.5-78)^{i}$ & $7(6-8)$ & $13(10-16)$ & & & & & & & $\begin{array}{c}\text { MRI-ASPECTS } \\
(4-6,7-10)\end{array}$ \\
\hline
\end{tabular}

Abbreviations: A, anterior circulation; A-P, anterior and posterior circulation (number of posterior circulation); ASPECTS, Alberta Stroke Program Early CT Scale; CT, computed tomography; iA, intra-arterial thrombolysis; ICA, internal carotid artery; iv tPA; intravenous tissue plasminogen activator infusion; LAO, large artery occlusion; MCA. Middle cerebral artery; N, No; NA, not applicable; NIHSS, National Institutes of Health Stroke Scale; NR, not reported; SD, standard deviation; TICI, Thrombolysis in Cerebral Infarction; TIMI, Thrombolysis in Myocardial Infarction; $Y$, yes

a: Among 51 patients who were enrolled in this study, follow up mRS were available in 48 patients. The data of 'characteristics of patients' was collected from 51 patients.

b: Odds ratio was adjusted.

c: The information of characteristics of subjects were extracted from the per protocol population (165 and 150). Subjects which used in meta-analysis are provided in ancillary analysis including protocol violators and excluding follow-up loss.

$\mathrm{d}$ : Among 103 patients who were enrolled in this study, number of successful recanalization and ICA occlusion were available in 102 patients.

e: In DEFUSE 2 trial, Merci device, Penumbra system, and intra-arterial tPA were used to most of patients and only 6 stentrievers were used. Combinations of more than one endovascular therapy were used in $45 \%$.

$\mathrm{f}$. The information of characteristics of subjects were extracted from the per protocol population (98 and 98)

g. median (range)

h. mean (standard deviation)

i. median and interquartile range (IQR)

j. 9 protocol violators $(0 \sim 5)$ included, 
Table 3. Risk Assessment of Individual Studies Using the National Institute of Health Quality Assessment Tool for Observational Cohort and Cross-sectional Studies

\begin{tabular}{|c|c|c|c|c|c|c|c|c|c|c|c|c|c|c|c|}
\hline STUDY & Q1 & Q2 & Q3 & Q4 & Q5 & Q6 & Q7 & Q8 & Q9 & Q10 & Q11 & Q12 & Q13 & Q14 & Quality Rating \\
\hline Hill' ${ }^{18}$ (PROACT-II) & + & + & + & + & - & - & + & + & + & NA & + & $C D$ & + & + & Good \\
\hline Imai $^{19}$ & + & + & NA & + & - & - & + & + & + & NA & + & CD & + & + & Fair \\
\hline Psycholgios $^{20}$ & + & + & NA & + & - & - & + & + & + & NA & - & CD & + & + & Fair \\
\hline $\mathrm{Hill}^{21}$ (IMS-III) & + & + & + & + & + & - & + & + & + & NA & + & CD & + & - & Good \\
\hline Inoue $^{22}$ & + & + & NA & + & - & - & + & + & + & NA & + & CD & + & + & Fair \\
\hline Yoo"1 (Penumbra Pivot) & + & + & + & + & - & - & + & + & + & NA & + & CD & + & - & Fair \\
\hline Berkhemer ${ }^{1}$ (MR-CLEAN) & + & + & + & + & - & + & + & + & + & NA & + & $\mathrm{CD}$ & + & + & Good \\
\hline Espinosa de Rueda ${ }^{12}$ & + & + & + & + & - & - & + & + & + & NA & + & CD & + & + & Good \\
\hline Goyal $^{3}$ (ESCAPE) & + & + & + & + & + & + & + & + & + & NA & + & CD & + & + & Good \\
\hline Jovin ${ }^{4}$ (REVASCAT) & + & + & - & + & + & + & + & + & + & NA & + & $C D$ & + & + & Good \\
\hline McTarggart ${ }^{23}$ (DEFUSE-II) & + & - & + & + & - & - & + & + & + & NA & + & CD & + & + & Fair \\
\hline Saver $^{5}$ (SWIFT-PRIME) & + & + & + & + & NA & + & + & + & + & + & + & CD & + & - & Good \\
\hline Spiotta $^{13}$ & + & - & NA & + & - & - & + & + & + & NA & + & $\mathrm{CD}$ & + & - & Fair \\
\hline $\mathrm{Kim}^{24}$ & + & + & + & + & NA & - & + & + & + & NA & + & $\mathrm{CD}$ & + & - & Fair \\
\hline
\end{tabular}

+, Yes; -, No; CD, cannot determine; NA, not applicable

statistics, $\left.\mathrm{P}=0.74 ; \mathrm{I}^{2}=0.00 \%\right)$ and six retrospective case series $(\mathrm{OR}=2.422 ; 95 \% \mathrm{CI}, 1.764$ to $3.326 ; \mathrm{P}<0.0001$; Q-statistics, $\mathrm{P}<0.05 ; \mathrm{I}^{2}=64.37 \%$ ) also demonstrated favorable outcomes in favor of high ASPECTS (Fig. 2A). There were no significant differences between subgroups (Q-statistics, $\mathrm{P}=0.567$ ). Subgroup analyses of four studies that included intra-arterial thrombolysis as the dominant endovascular treatment option also showed that initial high ASPECTS was associated with stronger odds for good outcomes $(\mathrm{OR}=2.638 ; 95 \% \mathrm{CI}$, 11.971 to $3.531 ; \mathrm{P}<0.0001$; Q-statistics, $\mathrm{P}=0.06$; $\mathrm{I}^{2}=59.67 \%$ ). Pooled analyses of eight studies that used new generation devices (suction thrombectomy and stent retriever) for thrombectomy yielded the same results $(\mathrm{OR}=2.074 ; 95 \% \mathrm{CI}, 1.580$ to $2.721 ; \mathrm{P}<0.0001$; $\mathrm{Q}$-statistics, $\mathrm{P}=0.41 ; \mathrm{I}^{2}=2.06 \%$ ). There were no significant differences between the subgroups (Q-statistics, $\mathrm{P}=0.236$ ) (Fig. 2C).

Meta-regression analysis demonstrated that the log odds ratios between high and low ASPECTS arms decreased with increases in the ICA occlusion rate (regression coefficient $=-0.048 ; 95 \%$ CI, -0.078 to $0.017 ; \mathrm{P}=0.002$ ) (Fig. 2D) in 11 studies, but did not identify any correlations with functional outcome on the basis of time to recanalization or the rate of preinterventional IVT.
Exclusion sensitivity analysis demonstrated that pooled estimates did not change with the exclusion of any one study for either effect size measure. Pooled estimates of good outcomes were not significantly different, with the exclusion of three studies that used MRI to score ASPECTS, four studies that did not have upper age limits for inclusion, and two studies that excluded subjects with ICA occlusions.

\section{Secondary outcomes}

Mortality was extracted from 1,085 patients of seven studies, and sICH was from 1,235 patients of eight studies. Mortality and sICH were significantly lower $(\mathrm{OR}=2.178$; 95\% CI, 1.530 to $3.100 ; \mathrm{P}<0.0001$; Qstatistics, $\mathrm{P}=0.32 ; \mathrm{I}^{2}=14.17 \%$; and $\mathrm{OR}=2.133 ; 95 \% \mathrm{CI}$, 1.510 to $3.015 ; \mathrm{P}<0.0001$; Q-statistics, $\mathrm{P}=0.35$; $\mathrm{I}^{2}=10.59 \%$ ) (Fig. $\left.3 \mathrm{~A}, \mathrm{~B}\right)$ in high ASPECTS than low ASPECTS.

In four studies, dichotomization of ASPECTS as very low $(\leq 4)$ versus intermediate to high $(>4)$ was available for a total of 1,062 patients. Meta-analysis demonstrated good outcomes in favor of intermediate to high ASPECTS (OR=4.077; 95\% CI, 1.884 to 8.825; $\mathrm{P}<0.0001$; Q-statistics, $\left.\mathrm{P}=0.05 ; \mathrm{I}^{2}=60.75 \%\right)$. Dichotomization into intermediate (5 or 6-7) and high ASPECTS (8-10) was available in nine studies. Meta- 


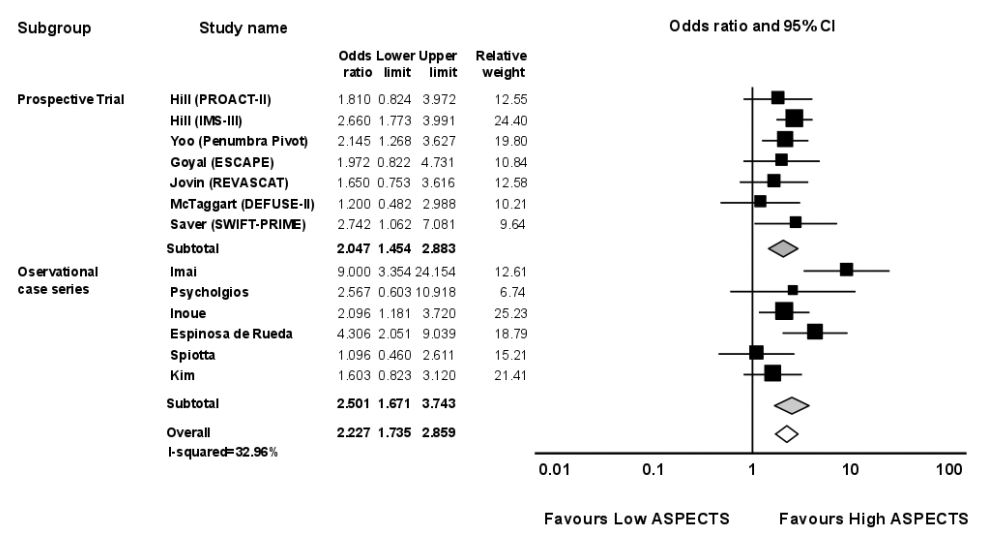

Funnel Plot of Standard Error by Log odds ratio

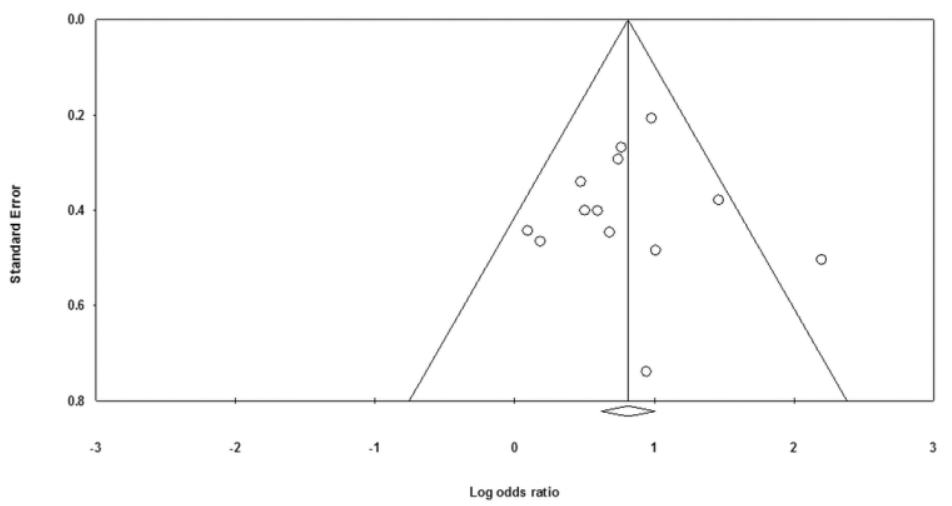

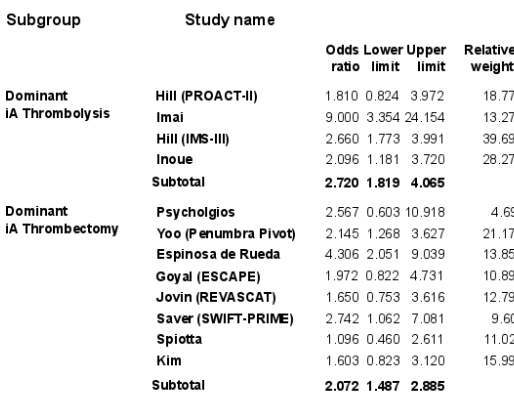

Fig. 2. Clinical outcomes of endovascular treatments in ischemic stroke patients with high ASPECTS vs. Iow ASPECTS A. Meta-analyses of high ASPECTS ( $>7)$ vs. low ASPECTS $(\leq 7)$ for favorable clinical outcome (modified Rankin scale score $0-2)(\mathbf{A})$. The relative size of the data markers indicates the weight of each study's sample size. The included trials for functional outcome are divided into subgroups according to study design (prospective randomized controlled or single-arm trials and observational case series). B. A funnel plot with all points A evenly distributed on both sides of the solid vertical line indicates no publication bias. C. Subgroup analysis of favorable outcomes of endovascular treatments in ischemic stroke patients with high ASPECTS vs. Iow ASPECTS. The included trials for functional outcome are divided into subgroups according to the methods of endovascular therapy (dominant intraarterial thrombolysis and dominant intraarterial thrombectomy). One study (23) which did not clearly present the endovascular methods was excluded from subgroup analysis for endovascular methods. D. Scatterplots of the relationship between the rate of ICA occlusion and log odds ratio. The

B size of the bubbles indicates the weight of each study in the meta-analysis. The trend line indicates the degree to which the log odds ratio decreases as the rate of ICA occlusion in subjects increases.

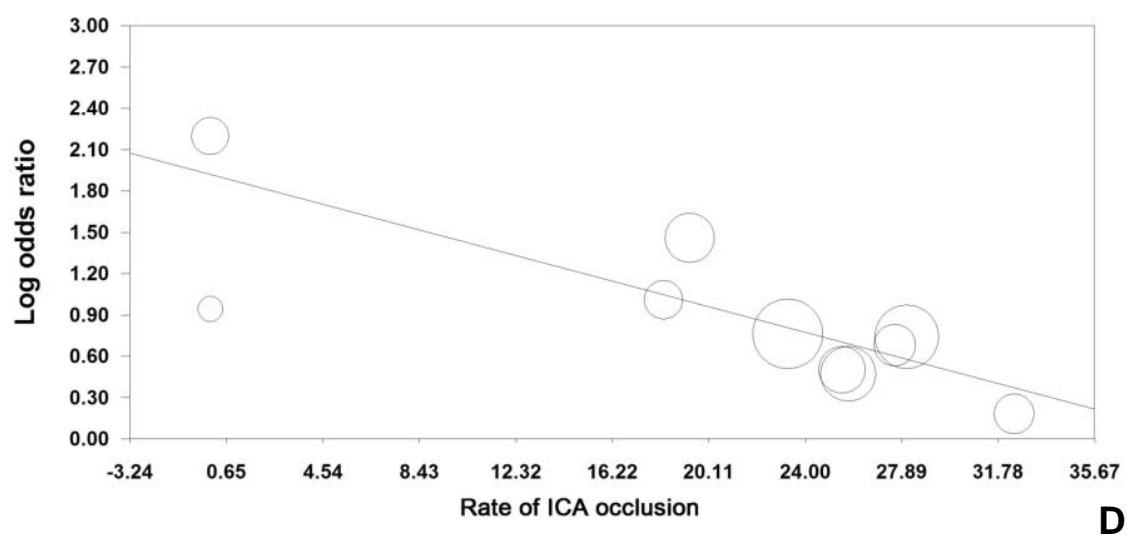


Study name

\begin{tabular}{|c|c|c|c|}
\hline & $\begin{array}{r}\text { Odds } \\
\text { ratio }\end{array}$ & $\begin{array}{r}\text { Lower } \\
\text { limit }\end{array}$ & $\begin{array}{r}\text { Upper } \\
\text { limit }\end{array}$ \\
\hline Hill (PROACT-II) & 1.184 & 0.487 & 2.877 \\
\hline Psycholgios & 6.533 & 1.410 & 30.268 \\
\hline Inoue & 2.169 & 1.128 & 4.170 \\
\hline Yoo (Penumbra Pivot) & 2.539 & 1.428 & 4.514 \\
\hline Goyal (ESCAPE) & 1.450 & 0.374 & 5.625 \\
\hline Spiotta & 4.122 & 1.545 & 10.594 \\
\hline Kim & 1.393 & 0.571 & 3.398 \\
\hline Overall & 2.178 & 1.530 & 3.100 \\
\hline
\end{tabular}

I-squared $=14.17 \%$
Odds ratio and $95 \% \mathrm{Cl}$

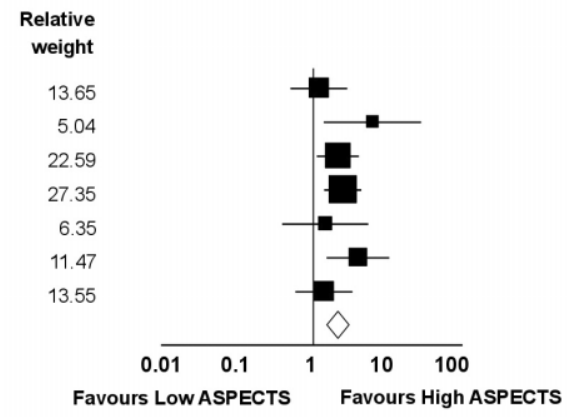

A
Study name

\begin{tabular}{|c|c|c|c|}
\hline & $\begin{array}{r}\text { Odds } \\
\text { ratio }\end{array}$ & $\begin{array}{r}\text { Lower } \\
\text { limit }\end{array}$ & $\begin{array}{r}\text { Upper } \\
\text { limit }\end{array}$ \\
\hline Hill (PROACT-II) & 1.184 & 0.487 & 2.877 \\
\hline Inoue & 3.931 & 1.123 & 13.757 \\
\hline Yoo (Penumbra Pivot) & 2.539 & 1.428 & 4.514 \\
\hline Goyal (ESCAPE) & 1.450 & 0.374 & 5.625 \\
\hline Jovin (REVASCAT) & 1.464 & 0.626 & 3.425 \\
\hline Saver (SWIFT-PRIME) & 4.400 & 1.204 & 16.084 \\
\hline Spiotta & 4.122 & 1.545 & 10.994 \\
\hline Kim & 1.393 & 0.571 & 3.398 \\
\hline Overall & 2.133 & 1.510 & 3.015 \\
\hline
\end{tabular}

Study name

$\begin{array}{lrrr} & \begin{array}{c}\text { Odds } \\ \text { ratio }\end{array} & \begin{array}{c}\text { Lower } \\ \text { limit }\end{array} & \begin{array}{r}\text { Upper } \\ \text { limit }\end{array} \\ \text { Hill (IMS-III) } & 3.290 & 1.653 & 6.550 \\ \text { Inoue } & 5.626 & 2.572 & 12.304 \\ \text { Yoo (Penumbra Pivot) } & 14.653 & 3.444 & 62.338 \\ \text { Kim } & 1.282 & 0.390 & 4.210 \\ \text { Overall } & \mathbf{4 . 0 7 7} & \mathbf{1 . 8 8 4} & \mathbf{8 . 8 2 5} \\ \text { I-square=60.75\% } & & & \end{array}$

Study name

$\begin{array}{lrrr}\text { Study name } & & & \\ & \begin{array}{r}\text { Odds } \\ \text { ratio }\end{array} & \begin{array}{c}\text { Lower } \\ \text { limit }\end{array} & \begin{array}{r}\text { Upper } \\ \text { limit }\end{array} \\ \text { Imai } & 9.000 & 3.354 & 24.154 \\ \text { Psycholgios } & 3.575 & 0.679 & 18.813 \\ \text { Hill (IMS-III) } & 2.224 & 1.424 & 3.475 \\ \text { Inoue } & 1.212 & 0.646 & 2.273 \\ \text { Yoo (Penumbra Pivot) } & 1.292 & 0.737 & 2.267 \\ \text { Jovin (REVASCAT) } & 1.650 & 0.753 & 3.616 \\ \text { McTaggart (DEFUSE-II) } 1.100 & 0.409 & 2.962 \\ \text { Saver (SWIFT-PRIME) } & 2.742 & 1.062 & 7.081 \\ \text { Kim } & 1.328 & 0.711 & 2.479 \\ \text { Overall } & \mathbf{1 . 8 8 0} & \mathbf{1 . 3 0 2} & \mathbf{2 . 7 1 3} \\ \text { I-Square=53.62\% } & & & \end{array}$

Fig. 3. Mortality and symptomatic intracranial hemorrhage of endovascular treatments in ischemic stroke patients with high ASPECTS vs. low ASPECTS.

A. Mortality at 90 days.

B. Symptomatic intracranial hemorrhage.
Odds ratio and $95 \% \mathrm{Cl}$

Relative weight

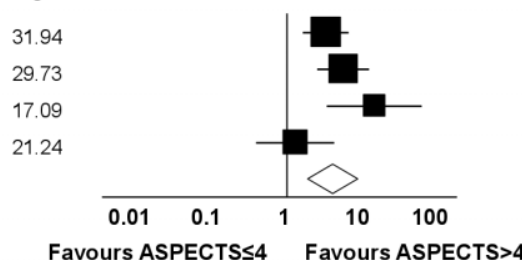

Favours ASPECTS $\leq 4$ Favours ASPECTS $>4$

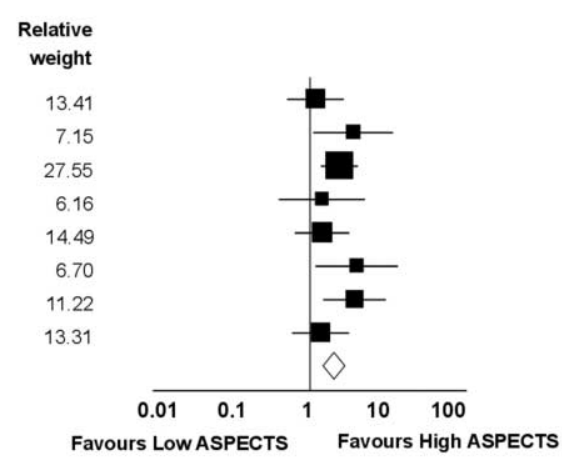

B

\section{A}

Fig. 4. Comparison among very low, intermediate, and high ASPECTS.

A. Favorable outcome (modified Rankin scale score $0-2$ at 90 days) of endovascular treatments in ischemic stroke patients with ASPECTS $\leq 4$ vs. ASPECTS $>4$.

B. Favorable outcome of endovascular treatments in ischemic stroke patients with intermediate ASPECTS (5 or 6 to 7 ) vs. high ASPECTS (8-10).
Odds ratio and $95 \% \mathrm{Cl}$

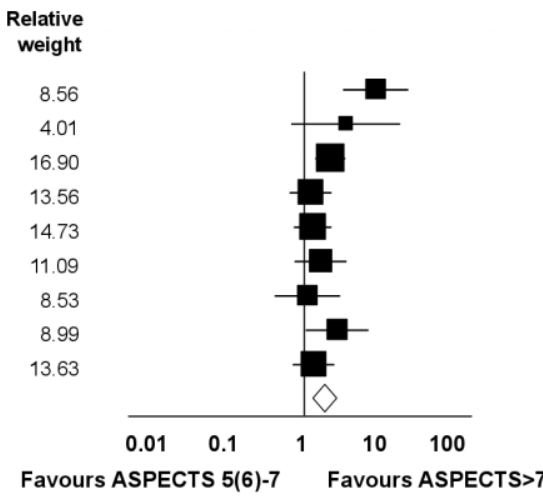


analysis demonstrated good outcomes in favor of high ASPECTS (OR $=1.880 ; 95 \% \mathrm{CI}, 1.3025$ to 2.713 ; $\mathrm{P}<0.0001$; Q-statistics, $\mathrm{P}<0.05 ; \mathrm{I}^{2}=53.62 \%$ ) (Fig. 4).

\section{DISCUSSION}

The meta-analyses showed that a high ASPECTS favored a good clinical outcome after endovascular therapy in comparison with a low ASPECTS. This supports that baseline ASPECTS is a reliable predictor of prognosis in patients with AIS undergoing endovascular therapy, as noted in several individual studies [18, $21,25]$. Additional subgroup and sensitivity analyses revealed that the primary endpoint of pooled data was not affected by potential biases, such as study design, imaging modality, or endovascular therapy method.

Because the baseline infarction volume is an essential factor affecting clinical results in AIS along with the time-window [26], many researchers have used ASPECTS to assess the efficiency of the treatment modality. In IVT of AIS, the prognostic value of ASPECTS was established in clinical trials with large cohorts, showing a linear correlation between ASPECTS and prognosis [10]. Dichotomous ASPECTS categories were also independent predictors of functional outcomes and sICH [15]. However, ASPECTS was not proven to modify the effectiveness of IVT in terms of improved functional outcomes in two randomized trials compared to placebo [27, 28], which does not support the exclusion of patients from IVT based on ASPECTS.

Unlike IVT, the present study indicates that ASPECTS can identify candidates who are suited for endovascular therapy in AIS and can help determine the best treatment option without delaying therapy. In this meta-analysis, the pooled data showed heterogeneity across enrolled studies, which was explained by treatment option, intraarterial thromobolysis versus intraarterial thrombectomy. Such differences in endovascular method have also been suggested to be a confounding factor in previous meta-analyses comparing the effectiveness of endovascular therapy vs. medical treatment of ischemic stroke $(29,30)$. Because the present analysis used a dichotomous classification of ASPECTS and many of enrolled studies did not provide cut-off values of ASPECTS for candidates of endovascular therapy but instead used the one-third rule, we were unable to specify the lowest ASPECTS value associated with therapeutical benefits after endovascular therapy. Therefore, further studies should be conducted to identify cut-off values of ASPECTS for selecting patients likely to benefit from endovascular therapy.

Although ASPECTS was originally designed for use with non-enhanced brain CT, recent studies assessed its practicability with advanced brain imaging methods, such as perfusion CT and MRI, due to limitations associated with interrater reliability, predictability, and sensitivity for early ischemic lesions when assessed using CT-ASPECTS compared with MRI-ASPECTS [23]. However, CT has the advantage of being an easy approach and saving the time to treatment, and has stronger evidence through many clinical trials. Therefore, CT-ASPECTS will not be replaced by alternative scoring systems in clinical practice within the next few years. The lower reliability of CT ASPECTS should be overcome by training clinicians who assess patients initially after admission to the hospital [31]. In this study, meta-regression showed that the rate of ICA occlusion was negatively correlated with odds ratios in favor of high ASPECTS. This finding suggests that lower recanalization rate, procedural difficulty, and weaker collateral supply in ICA occlusion are factors that decrease the likelihood of good outcomes, despite initial small volumes of infarction [32].

Our study has several potential limitations. First, because baseline ASPECTS was not randomized by researchers in most studies, this manuscript was described based on guidelines for meta-analyses of observational studies. Second, our results could be constrained by the unclear risk of bias owing to incomplete data in a few studies.

Our meta-analysis of pooled data, including prospective trials and observational studies, provides high-level evidence of outcome prognostication of dichotomized ASPECTS in endovascular therapy for ischemic stroke. Scoring the baseline ASPECTS would enhance the selection of candidates for endovascular therapy by predicting prognosis in the management of ischemic stroke.

\section{Acknowledgement}

We thank Dr. Woong Yoon for providing raw data referenced in his article (ref. 24).

\section{References}

1. Berkhemer OA, Fransen PS, Beumer D, van den Berg LA, Lingsma HF, Yoo AJ, et al. A randomized trial of intraarterial treatment for acute ischemic stroke. $N$ Engl J Med 2015;372:1120

2. Campbell BC, Mitchell PJ, Kleinig TJ, Dewey HM, Churilov L, 


\section{Alberta Stroke Program Early CT Score in the Prognostication after Endovascular Treatment for Ischemic Stroke}

Yassi N, et al. Endovascular therapy for ischemic stroke with perfusion-imaging selection. N Engl J Med 2015;372:1009-1018

3. Goyal M, Demchuk AM, Menon BK, Eesa M, Rempel JL, Thornton J, et al. Randomized assessment of rapid endovascular treatment of ischemic stroke. N Engl J Med 2015;372:1019-1030

4. Jovin TG, Chamorro A, Cobo E, de Miquel MA, Molina CA, Rovira A, et al. Thrombectomy within 8 hours after symptom onset in ischemic stroke. N Engl J Med 2015;372:2296-2306

5. Saver JL, Goyal M, Bonafe A, Diener HC, Levy EI, Pereira VM, et al. Stent-retriever thrombectomy after intravenous t-PA vs. t-PA alone in stroke. N Engl J Med 2015;372:2285-2295

6. Larrue V, von Kummer R, del Zoppo G, Bluhmki E. Hemorrhagic transformation in acute ischemic stroke. Potential contributing factors in the European Cooperative Acute Stroke Study. Stroke 1997;28:957-960

7. von Kummer R, Allen KL, Holle R, Bozzao L, Bastianello S, Manelfe C, et al. Acute stroke: usefulness of early CT findings before thrombolytic therapy. Radiology 1997;205:327-333

8. Fiorelli M, Bastianello S, von Kummer R, del Zoppo GJ, Larrue $\mathrm{V}$, Lesaffre E, et al. Hemorrhagic transformation within 36 hours of a cerebral infarct: relationships with early clinical deterioration and 3-month outcome in the European Cooperative Acute Stroke Study I (ECASS I) cohort. Stroke 1999;30:2280-2284

9. Pexman JH, Barber PA, Hill MD, Sevick RJ, Demchuk AM, Hudon ME, et al. Use of the Alberta Stroke Program Early CT Score (ASPECTS) for assessing CT scans in patients with acute stroke. AJNR Am J Neuroradiol 2001;22:1534-1542

10. Hill MD, Buchan AM, Canadian Alteplase for Stroke Effectiveness Study I. Thrombolysis for acute ischemic stroke: results of the Canadian Alteplase for Stroke Effectiveness Study. CMAJ 2005;172:1307-1312

11. Yoo AJ, Zaidat OO, Chaudhry ZA, Berkhemer OA, Gonzalez $\mathrm{RG}$, Goyal M, et al. Impact of pretreatment noncontrast CT Alberta Stroke Program Early CT Score on clinical outcome after intra-arterial stroke therapy. Stroke 2014;45:746-751

12. Espinosa de Rueda M, Parrilla G, Manzano-Fernandez S, GarciaVillalba B, Zamarro J, Hernandez-Fernandez F, et al. Combined Multimodal Computed Tomography Score Correlates With Futile Recanalization After Thrombectomy in Patients With Acute Stroke. Stroke 2015;46:2517-2522

13. Spiotta AM, Vargas J, Hawk H, Turner R, Chaudry MI, Battenhouse $\mathrm{H}$, et al. Impact of the ASPECT scores and distribution on outcome among patients undergoing thrombectomy for acute ischemic stroke. J Neurointerv Surg 2015;7:551-558

14. Stroup DF, Berlin JA, Morton SC, Olkin I, Williamson GD, Rennie D, et al. Meta-analysis of observational studies in epidemiology: a proposal for reporting. Meta-analysis Of Observational Studies in Epidemiology (MOOSE) group. JAMA 2000;283: 2008-2012

15. Barber PA, Demchuk AM, Zhang J, Buchan AM. Validity and reliability of a quantitative computed tomography score in predicting outcome of hyperacute stroke before thrombolytic therapy. ASPECTS Study Group. Alberta Stroke Programme Early CT Score. Lancet 2000;355:1670-1674

16. Seyyedi S, Cengiz K, Kamasak M, Yildirim I. An object-oriented simulator for 3D digital breast tomosynthesis imaging system. Comput Math Methods Med 2013;2013:250689

17. Higgins JP, Altman DG, Gotzsche PC, Juni P, Moher D, Oxman $\mathrm{AD}$, et al. The Cochrane Collaboration's tool for assessing risk of bias in randomised trials. BMJ 2011;343:d5928

18. Hill MD, Rowley HA, Adler F, Eliasziw M, Furlan A, Higashida $\mathrm{RT}$, et al. Selection of acute ischemic stroke patients for intraarterial thrombolysis with pro-urokinase by using ASPECTS. Stroke 2003;34:1925-1931

19. Imai K, Mori T, Izumoto H, Watanabe M, Kunieda T, Takabatake $\mathrm{N}$, et al. MR imaging-based localized intra-arterial thrombolysis assisted by mechanical clot disruption for acute ischemic stroke due to middle cerebral artery occlusion. AJNR Am J Neuroradiol 2011;32:748-752

20. Psychogios MN, Schramm P, Frolich AM, Kallenberg K, Wasser K, Reinhardt L, et al. Alberta Stroke Program Early CT Scale evaluation of multimodal computed tomography in predicting clinical outcomes of stroke patients treated with aspiration thrombectomy. Stroke 2013;44:2188-2193

21. Hill MD, Demchuk AM, Goyal M, Jovin TG, Foster LD, Tomsick TA, et al. Alberta Stroke Program early computed tomography score to select patients for endovascular treatment: Interventional Management of Stroke (IMS)-III Trial. Stroke 2014;45:444-449

22. Inoue M, Olivot JM, Labreuche J, Mlynash M, Tai W, Albucher JF, et al. Impact of diffusion-weighted imaging Alberta stroke program early computed tomography score on the success of endovascular reperfusion therapy. Stroke 2014;45:1992-1998

23. McTaggart RA, Jovin TG, Lansberg MG, Mlynash M, Jayaraman $\mathrm{MV}$, Choudhri OA, et al. Alberta stroke program early computed tomographic scoring performance in a series of patients undergoing computed tomography and MRI: reader agreement, modality agreement, and outcome prediction. Stroke 2015;46:407-412

24. Kim SK, Yoon W, Park MS, Heo TW, Baek BH, Lee YY. Outcomes Are Not Different between Patients with Intermediate and High DWI-ASPECTS after Stent-Retriever Embolectomy for Acute Anterior Circulation Stroke. AJNR Am J Neuroradiol 2016

25. Goyal M, Menon BK, Coutts SB, Hill MD, Demchuk AM. Effect of baseline CT scan appearance and time to recanalization on clinical outcomes in endovascular thrombectomy of acute ischemic strokes. Stroke 2011;42:93-97

26. Schriger DL, Kalafut M, Starkman S, Krueger M, Saver JL. Cranial computed tomography interpretation in acute stroke: physician accuracy in determining eligibility for thrombolytic therapy. JAMA 1998;279:1293-1297

27. Dzialowski I, Hill MD, Coutts SB, Demchuk AM, Kent DM, Wunderlich O, et al. Extent of early ischemic changes on computed tomography (CT) before thrombolysis: prognostic value of the Alberta Stroke Program Early CT Score in ECASS II. Stroke 2006;37:973-978

28. Demchuk AM, Hill MD, Barber PA, Silver B, Patel SC, Levine $\mathrm{SR}$, et al. Importance of early ischemic computed tomography changes using ASPECTS in NINDS rtPA Stroke Study. Stroke 2005;36:2110-2115

29. Badhiwala JH, Nassiri F, Alhazzani W, Selim MH, Farrokhyar F, Spears J, et al. Endovascular Thrombectomy for Acute Ischemic Stroke: A Meta-analysis. JAMA 2015;314:1832-1843

30. Chen CJ, Ding D, Starke RM, Mehndiratta P, Crowley RW, Liu $\mathrm{KC}$, et al. Endovascular vs medical management of acute ischemic stroke. Neurology 2015;85:1980-1990

31. Coutts SB, Hill MD, Demchuk AM, Barber PA, Pexman JH, Buchan AM. ASPECTS reading requires training and experience. Stroke 2003;34:e179; author reply e179

32. Nogueira RG, Liebeskind DS, Sung G, Duckwiler G, Smith WS, 
Chang-Woo Ryu, et al.

Merci, et al. Predictors of good clinical outcomes, mortality, and successful revascularization in patients with acute ischemic stroke undergoing thrombectomy: pooled analysis of the Mechanical
Embolus Removal in Cerebral Ischemia (MERCI) and Multi MERCI Trials. Stroke 2009;40:3777-3783 